\title{
MAGNETIC FIELDS AND THE DYNAMICS OF MOLECULAR CLOUDS
}

\author{
J.L. PUGET \\ Institut d'Astrophysique Spatiale, \\ Bat. 120, Université Paris Sud \\ F-91405, Orsay, France
}

\begin{abstract}
Magnetic fields are believed to play an important role in the star formation process. Correlations in the velocity field in molecular filaments are indicative of dynamical interactions between clouds and parts within a cloud. The magnetic field is a likely candidate as the vector of such interactions. Perturbations of the field at large scales can feed the velocity dispersion within condensations at small scale. This mechanism is discussed in the framework of two simple analytical approximations describing transverse waves fed into plane parallel slabs.

KEYWORDS: Molecular clouds evolution; Alfvèn Waves; Magnetic field; Filamentary structure; Turbulence .
\end{abstract}

\section{INTRODUCTION}

Interstellar gas is cycled through a system which can be very schematically described by a three steps cycle (molecular clouds are observed in these different states, the evolution from the one to the next is only a reasonable speculation at this stage).

1- a large system of low density gas and HI clouds in quasi pressure equilibrium undergoes a gravitational and/or thermal instability (Elmegreen 1985, Lioure and Chièze 1990) and gathers into a marginally bound complex.

2- the large scale kinetic energy in this complex is dissipated and the complex slowly contracts. This stage is best illustrated by large quiescent molecular complexes observed in the solar vicinity like the one observed by Maddalena and Thaddeus (1985) or Pérault et al. (1985).

Low mass stars are forming within the densest molecular clouds in such a complex. The Taurus cloud belongs to this phase.

3- as more kinetic energy is dissipated, the central part of the complex becomes more condensed and more and more massive stars are formed in this core. The $\rho$ Ophiuchus and the Orion molecular clouds belong to this third phase.

In this last phase, massive stars produce strong winds, HII regions and supernovae.Part of the central core is photodissociated and the complex is dispersed. In this phase the potential energy of the whole cloud system is restored to be dissipated in the next contraction phase.

Low mass stars also have strong winds and outflows during the $\mathrm{T}$ Tauri phase and also inject in the complex an amount of kinetic energy which could be non negligible in some phases but smaller than that coming from massive stars (Puget 1985). 
The large scale kinetic energy plays an essential role in the equilibrium of the complex at least on large scales. It probably also contributes to create density structures as the flows are highly supersonic and thus compressible. The star formation process cannot be described by a simple Jeans analysis of gravitational instability in a uniform medium where thermal pressure is the main force acting against gravity. Bonazzola et al. (1987, 1990) have discussed some of the complicated processes which control star formation when the supersonic turbulence might stabilize the large scales more than the small scales and at the same time creates density fluctuations. In such a system the star formation process is not any more controlled by local parameters (like temperature, gas density, magnetic field strength, and ionization state), but also, and possibly mainly, by the dynamical state of the gas which depends, through turbulent cascades, on the large scale environment of the cloud considered

It is thus essential to understand the physical mechanisms controlling this turbulent cascade in the interstellar medium to learn what controls the star formation rate and the initial mass function.

It has been stressed for several years that the magnetic field has an intensity such that its dynamical role is probably important. Recent observations have confirmed this point of view (see for example the review by Heiles at this conference).

The role of the static magnetic field on the gravitational stability of cold interstellar gas has been studied in detail. Secular evolution through ambipolar diffusion has also been studied and dynamical effects during the collapse phase to transfer out the angular momentum have also been addressed (see Mc Kee et al. 1991 for a review).

This paper concentrates on the less studied role of the magnetic field on the dynamics of the interstellar gas before any protostellar collapse takes place, and more specifically on the turbulent cascade in molecular complexes. We thus stress the role of the perturbations of the average field and the associated magneto hydrodynamical (MHD) waves on the stability of clouds more than the static role of the average field.

To separate factors as much as possible, we concentrate on complexes with low star formation rate or on part of complexes far away for massive stars.

\section{OBSERVATIONAL EVIDENCE}

The quasi virialized state of quiescent molecular complexes is well established. Furthermore the average density is low and thus the dense molecular material has a low volume filling factor in those complexes: 5 to $10 \%$ (Pérault et al., 1985).

Most of the volume of the complex is filled with rather low density gas likely to be warm HI (Falgarone and Puget, 1986). Nevertheless the molecular clouds do not behave in the complex like stars in a bound cluster. The clouds are gathered into substructures of the complex in a hierarchical structure in which no preferred scale emerges (Pérault et al 1986, Scalo 1987, Falgarone and Phillips this conference ). This is a strong indication that, despite the large density contrasts, the clouds in the complex interact strongly with each other. The large density contrast makes it very unlikely that they interact through pure hydrodynamical effects (in a supersonic turbulence large density contrasts can be generated but the dense phase contains only a small fraction of the total mass).

Correlations in both the spatial and the velocity distribution of molecular material in complexes is in fact directly observable in form of long filaments. Well known examples can be found in the Taurus-Auriga-Perseus complex (Ungerechts and 
Thaddeus, 1987), the $\rho$ Ophiuchus complex (Loren, 1989), the Northern Coalsack (Pérault et al., 1985), and L204 (Heiles 1988). These structures are very elongated. Along these filaments, the velocity is very well correlated, changing smoothly. The velocity gradient is much larger than the velocity dispersion of the individual clumps making the filament (see for example the case plotted in figure 1). This suggests that in these filaments a physical link maintains the spatial correlation to keep them from dispersing in a few million years. Pure hydrodynamical turbulence could create such correlations as long as the specific inertia in the surrounding medium is not small with respect to that of the filament itself, but this is probably not the case in the low density complexes.

The magnetic field is an obvious candidate to play that role. Nevertheless the direction of the field as revealed by star light polarization is not always that of the filament as for example in L204 (Heiles 1988). Neverless a wave pattern is present both in projection on the sky and velocity. We searched for similar patterns along other filaments (see also Fukui, this conference).

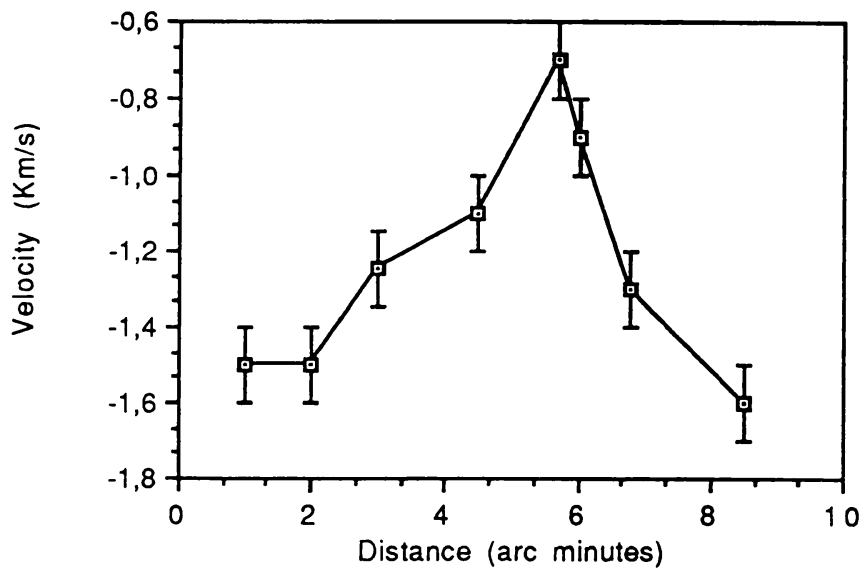

Figure 1: Radial velocity as a function of distance along the $\mathrm{C} 18 \mathrm{O}$ ridge At a distance $d=200 \mathrm{pc}, 1$ arc minute is $0.05 \mathrm{pc}$

Assuming that the field is strong enough to dynamically link efficiently the clouds together along a filament, we can extract the basic characteristics of transverse MHD waves along these filaments which would account for the velocities observed. The values obtained that way and reported in Figure 2 are interesting indicators but it should be remembered that the assumption of pure tranverse waves is certainly too simple.

To test further this hypothesis, Falgarone et al. (1991) have investigated the substructure of some of these filaments. In Auriga a dense ridge $0.1 \mathrm{pc}$ wide is observed in $\mathrm{C} 180$ which is aligned with the large filament (5 pc wide) in which it is embedded. Along this ridge several condensations $(0.02 \mathrm{pc}$ dense cores) can be identified. Figure 1 shows the radial velocity as function of distance along the ridge. This reinforces the idea that the magnetic field is needed even if the filaments are generated by a wind or a strong photon flux acting on the surface(Bally, this conference), because it shows that the inner small scale and the outer large scale are dynamically coupled.It is quite striking than the periods involved in these waves are not changing fast with scale within a given filament. 
Because projection effects affect the observed amplitudes of transverse waves in opposite directions for velocity and displacement, one must rely on the upper envelopes in Figure 2. They can be approximated by

$\Delta \mathrm{v}=1.22 \lambda_{\mathrm{pc}}^{0.2} \mathrm{~km} / \mathrm{s}$ and $\Delta \mathrm{l}=0.26 \lambda_{\mathrm{pc}}^{0.8} \mathrm{pc}$

leading to $\omega=4.75 \lambda_{\mathrm{pc}}^{-0.6} \mathrm{Myr}^{-1}$ and $\mathrm{v}_{\mathrm{A}}=0.75 \lambda_{\mathrm{pc}}^{0.4} \mathrm{~km} / \mathrm{s}$.

This behaviour of the Alfvèn velocity corresponds to a magnetic field of $7 \mu \mathrm{G}$ on large scale $\left(100 \mathrm{pc}, \mathrm{n}_{\mathrm{H} 2} \sim 5 \mathrm{~cm}^{-3}\right)$ and $20 \mu \mathrm{G}$ on the smallest scales $\left(0.1 \mathrm{pc}, \mathrm{n}_{\mathrm{H} 2} \sim 10^{4} \mathrm{~cm}^{-3}\right)$

\section{ROLE OF MAGNETIC FIELDS IN INTERSTELLAR TURBULENCE}

Clifford and Elmegreen (1983) and Arons and Max (1975) have shown that molecular clouds can interact through the magnetic field threading them and that this limits their mean free path. Falgarone and Puget (1986) have used the same analytical approach to compute the kinetic energy injected in a cloud by the magnetic interactions with its neighbours. They show that this mechanism generates trapped waves in the clouds with most of the power at scales comparable to the cloud size and with a level such that the waves are becoming non linear (perturbations of the field not very small compared to the average field).This stabilisation mechanism has been further investigated by Pudritz and Carlsberg (see Pudritz, this conference).

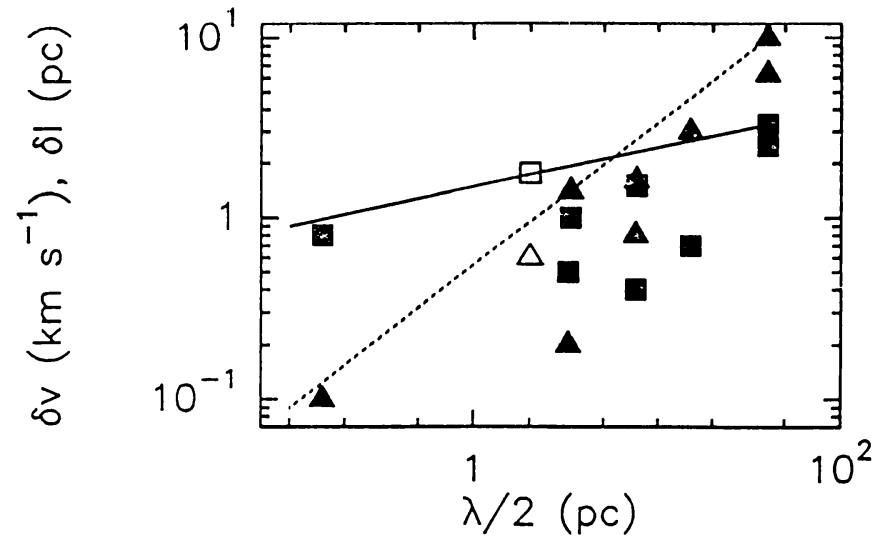

Figure 2: Amplitudes of waves along filaments as a function of half the wavelength. Triangles are velocities, squares are displacements. The full symbols refer to cases studied by Puget et al .(1991), the empty symbols are from Heiles (1988). The upper envelopes are given by the full line (displacement) and dashed line (velocity). 
This has two important consequences : it could explain how the turbulence is fed in molecular clouds even when no detectable star is forming within these clouds. Futhermore it leads to a field configuration in which the support against gravity in the direction of the average field might be comparable to the support in the direction perpendicular to it. Finally it efficiently feeds energy at scales smaller but comparable to the cloud size: the spectrum of the injected energy in the cloud is steep $\left(P(k)=k^{-\alpha}, \alpha>\right.$ 2). This is what is required to explain why small scales seem to be more unstable than large ones contrary to what the classical Jeans criterion would indicate (Bonazzola et al. 1987,1990).

The kinetic energy injected by this mechanism would cascade to smaller scales through non linear interactions and dissipate through wave steepening (long wavelengths) and ohmic dissipation (short wavelengths) (Zweibel and Josafatsson , 1983).

The resulting MHD turbulence in the cloud cannot be evaluated simply and requires numerical simulations.

As a first approach to the question of stability, Puget and Falgarone (1991) develop a very simple model to investigate how the energy injected scales with density.

The cloud can be modeled as a dense slab which receives a spectrum of transverse Alfvèn waves. If the ratio of the Alfvèn velocity inside the slab to the Alfvèn velocity outside is noted $\varepsilon=\left(\rho_{\text {in }} / \rho_{\text {out }}\right)^{-1 / 2}<<1$, the amplitude of the waves inside the slab (of thickness $d$ ) as a function of frequency shows resonances for :

$$
\omega=n \frac{\pi}{d} v_{A} \text { ( } n \text { being an integer) }
$$

Table 1 gives amplitude and energy density for resonant waves after integrating over frequency assuming a white incident spectrum. This shows that the energy density inside the slab is dominated by the resonant modes and that it increases with density like $1 / \varepsilon$. The finite size of the clouds is thus essential to the feeding mechanism (the energy fed a semi infinite medium decreases with density).

This very simple linear analysis suggests that a two dimensional slab can be stabilized by the wave energy fed into it by this mechanism.

The problem is of course much more complicated (it is non stationary, non linear). The spectrum of waves incident on a cloud certainly does not have a flat spectrum but more likely a steep spectrum.

The longest wavelengths just generate motions of the cloud as a whole. Only can the short wavelengths perturbations with smaller amplitude feed the inside of the slab in the linear regime. Falgarone and Puget (1986) suggest that in the non linear regime low frequency but large amplitude perturbations of the field will excite all the resonant modes with a spectrum steeper than $\mathrm{k}^{-2}$.

Of course a molecular cloud, even if it has sharp edges, does not have a simple geometry and thus no sharp resonant modes. Furthermore, density fluctuations will scatter and reflect the waves inside the slab ( $\mathrm{Li}$ and Zweibel, 1987). The large injection of energy at the resonant frequencies is due to the reflected waves from the other interface coming back with the right phase. In a realistic cloud geometry, there will not be plane reflected waves but there will still be perturbations of the field inside the cloud boundary due to reflected waves on other cloud boundaries and thus one might expect that the resonant peaks are washed out but that the integral of the injected energy is conserved. 
This can be checked to be true for multiple layers with different densities. The resonant pattern becomes much more complicated but in each slab the behaviour of the energy density of the waves with density remains those given in Table 1 (after averaging over frequency). A three dimensional MHD numerical simulation is needed to check this point under more general conditions.

In summary, the non linear waves generated in the cloud have large enough magnetic energy gradients to generate density fluctuations (see also B. Elmegreen at this conference). The question of the density and velocity dispersion distribution generated by non linear outside perturbations and the stability of the resulting configuration against gravitational collapse is an open question which requires three dimensional numerical simulations with a large dynamical range and non periodic boundary conditions in one dimension (Gispert, Puget, Bonazzola 1991).

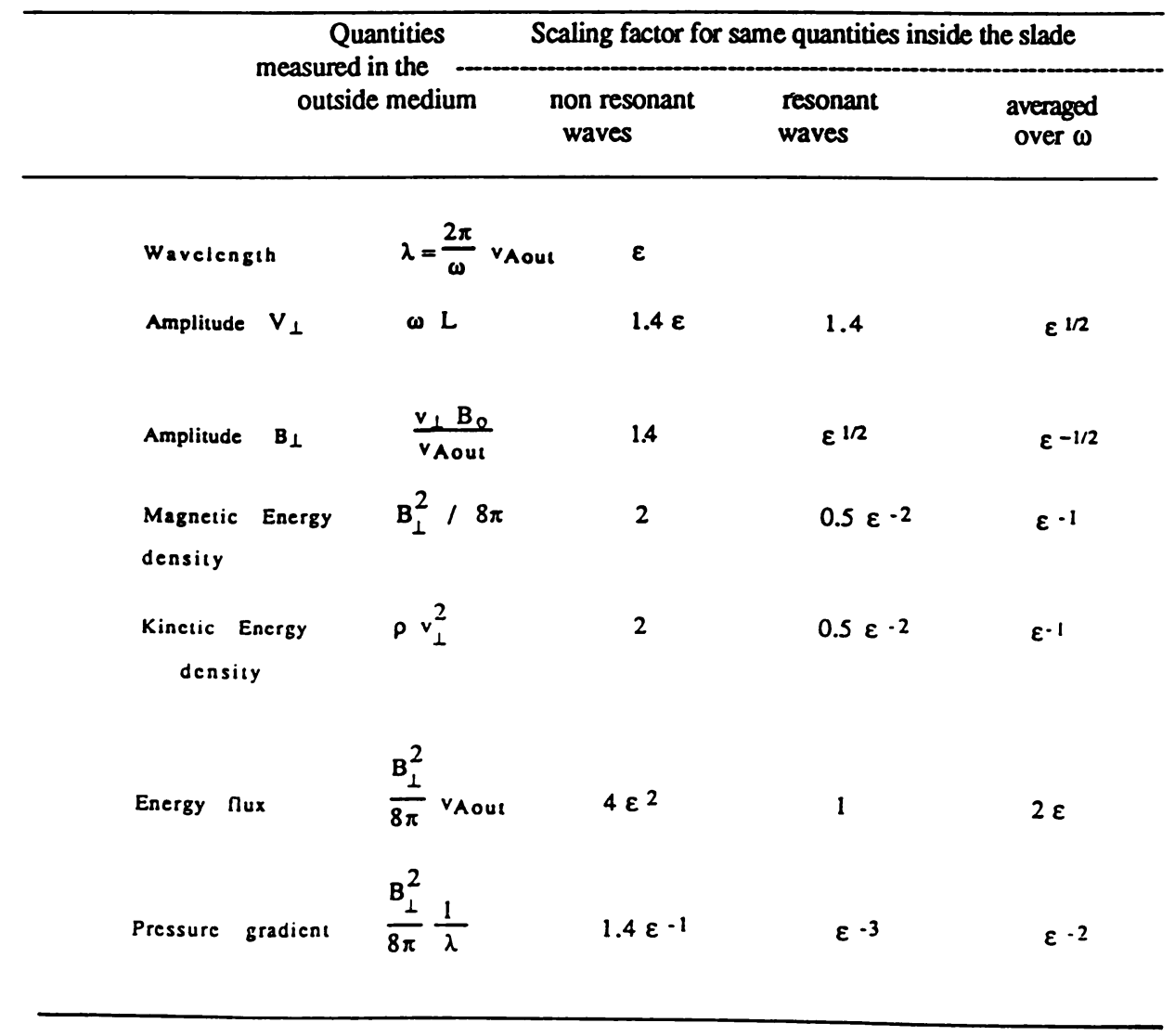

Table 1 


\section{REFERENCES:}

Arons J.and Max, C.E., 1975, Ap.J. Lett. 196,L77.

Bonazzola S., Falgarone E., Heyvaerts J., Pérault M., and Puget J.L. 1987, Astron. Astrophys. 172, 293

Bonazzola S., Pérault M., Puget J.L., Heyvaerts J., Falgarone E., 1990, submitted to Journal of Fluid Mechanics.

Clifford, P. and Elmegreen B.G. 1983, M. N. R. A. S. , 202,629

Elmegreen B.G. 1985, in Birth and infancy of stars ed. R. Lucas, A. Omont,and

R. Stora , North Holland Physics Pub.,p.215

Falgarone E., Puget J.L. 1986, Astron. Astrophys. 162,235

Falgarone E., Puget J.L., Pérault M., 1991, in preparation.

Gispert R., Puget J.L., Bonazzola S., 1991, in preparation.

Heiles C , 1988, Ap.J. 324,321

Li H.S. and Zweibel E.G., 1987, Ap.J. 322,248

Lioure A. and Chièze 1990, Astron. Astrophys. 235, 379.

Loren R.B. 1989, Ap.J. 338, 925.

Maddalena R.J., and Thaddeus P.,1985,Ap.J.,294,231.

McKee C., Zweibel E.G., Goodman A.A. and Heiles C., 1991, to be published in Protostars and Planets III.

Pérault M., Falgarone E. and Puget J.L. 1985, Astron. Astrophys., 152,371

Pérault M., Falgarone E. and Puget J.L. 1986, Astron. Astrophys.,157,139

Puget J.L., 1985, in Birth and infancy of stars, R.Lucas, A.Omont and R.Stora

Eds.,North Holland Physics Pub.

Puget J.L., Falgarone E., 1991, in preparation.

Scalo J.,1987, Interstellar Processes, eds. D.J. Hollenbach and H.A. Thronson.

Ungerechts, H. and Thaddeus, P., 1987, Ap. J. Suppl. 63,645.

Zweibel E.G., and Josafatsson K. 1983, Ap.J. 270,511 